\title{
Dual-hop Amplify-and-Forward Transmission with Imperfect Channel Estimates at the Relay
}

\author{
Zoran Hadzi-Velkov*, Diomidis S. Michalopoulos ${ }^{\dagger}$, George K. Karagiannidis ${ }^{\ddagger}$, and Robert Schober ${ }^{\dagger}$ \\ *Faculty of Electrical Engineering and Information Technologies, Ss. Cyril and Methodius University, Skopje \\ ${ }^{\dagger}$ Department of Electrical and Computer Engineering, University of British Columbia, Vancouver, Canada \\ ${ }_{\ddagger}^{\ddagger}$ Department of Electrical and Computer Engineering, Aristotle University of Thessaloniki, Thessaloniki
}

\begin{abstract}
With reference to the typical three-node amplify-andforward (AF) relaying setup, where a single relay assists the communication between a source terminal and a destination terminal, we investigate the effect of outdated channel state information (CSI) at the relay on the overall performance. In particular, we consider CSIassisted (i.e., variable-gain) AF relaying and derive expressions for the outage and the error probability for the case where the relay gain is adapted based on outdated estimates of the source-relay channel, when operating over Nakagami- $m$ fading. The obtained expressions are functions of the scale and shape fading parameters, as well as the correlation coefficient between the actual source-relay channel and its corresponding estimate. Numerical results reveal a considerable degradation of the overall performance, when CSI acquisition is not perfect.
\end{abstract}

\section{INTRODUCTION}

Wireless relaying technology serves as a major candidate for future wireless networks, such as the Long Term EvolutionAdvanced (LTE-A) system, due to a number of advantages that it promises in terms of system coverage and performance [1]. As a result, terrestrial networks are able to extend their coverage to distant areas by using relays, while the distributed nature of wireless relaying allows for combating not only small-scale fading, as conventional diversity techniques do, but also largescale fading.

Amplify and forward (AF) relaying, which refers to simple amplification and forwarding of the information sent from a source terminal to a destination terminal, is considered as the simplest implementation of wireless relaying in practice, since no other form of processing at the relay is required. Among the several variations of AF relaying, the most common one is socalled "CSI-assisted" (i.e., variable-gain) AF relaying, where the gain employed at the relay compensates for the instantaneous strength of the source-relay link, aiming at maintaining a constant relay transmit power. In order to do so, the relay relies on accurate monitoring of the source-relay channel, such that the amplification gain is modified according to the obtained channel state information (CSI). In this respect, the majority of the works in the literature have considered perfect CSI acquisition (see, e.g., [2][5] and the references therein). Of particular interest is the work in [5], where it was shown that the gain policy that compensates for the source-relay channel leads to optimal performance of CSIassisted AF relaying, provided that the available CSI at the relay is perfect.

In this regard, we relax here the assumption of perfect CSI acquisition at the relay, and examine the effect on the overall outage and error probability performance when operating over Nakagami- $m$ fading channels. To be more precise, this work investigates the performance of CSI-assisted AF relaying in scenarios where the relay obtains outdated CSI of the source-relay link. The outdated CSI acquisition applies to fast fading scenarios and stems from the time-varying nature of fading channels, such that the source-relay channel changes faster than the channel estimation process is repeated. It is noted that this is not the first time that outdated CSI is considered in the context of relay schemes (see, e.g., [6]-[8] and the references therein). However, in [6]-[8] it was assumed that the outdated channel estimation affects the relay selection process in multiple-relay setups, whereas in this work we consider the fundamental three node relaying setup, consisting of single source, a single relay and a single destination terminal, and concentrate on the effect of outdated CSI at the relay on the overall performance. Furthermore, in [6]-[8] the relay gain was assumed to be based on perfect CSI, whereas we assume here that it is calculated based on outdated CSI. By quantifying the level of CSI imperfection through the correlation coefficient, $\rho$, between the squared actual estimated channel values, expressions for the resulting outage and error probability are obtained. It is concluded that any deviation from the perfect CSI case (i.e., $\rho=1$ ) leads to a performance degradation, in terms of both outage and error probability, yet without affecting the slopes of the corresponding curves.

\section{System AND ChanNEL MODEL}

Let us consider a dual-hop wireless communication system with an AF relay, consisting of a source $S$, relay $R$, and destination $D$. The total instantaneous signal-to-noise ratio (SNR) at $D$ is given by

$$
\gamma=\frac{E_{S} \alpha^{2} \beta^{2} G^{2}}{\beta^{2} G^{2} N_{01}+N_{02}},
$$

where $E_{S}$ is the power of the transmitted signal from $S, N_{01}$ and $N_{02}$ are the powers of the additive white Gaussian noise (AWGN) at the relay and the destination, respectively, $\alpha$ and $\beta$ are the fading amplitudes of the $S-R$ and the $R-D$ links, respectively, and $G$ is the relay amplification factor. The fading amplitudes $\alpha$ and $\beta$ are assumed to follow the Nakagami- $m$ distribution with fading parameters $m_{X}$ and $m_{Y}$, respectively, and $E\left[\alpha^{2}\right]=\Omega_{X}$ and $E\left[\beta^{2}\right]=\Omega_{Y}$.

In the case of CSI-assisted AF relays, $G$ is selected according to $G=\sqrt{E_{R} /\left(E_{S} \alpha^{2}\right)}$ assuming relay $R$ perfectly estimates the fading amplitude of the $S-R$ link, $\alpha$. If the estimate is imperfect (i.e., relay $R$ estimates the fading amplitude of the $S-R$ link to 
be $\hat{\alpha}$, instead of $\alpha$ ), then $G$ is selected according to

$$
G^{2}=\frac{E_{R}}{E_{S} \hat{\alpha}^{2}}
$$

Combining (1) and (2), we obtain

$$
Z=\frac{X Y}{\hat{X}+Y}
$$

where $Z=\gamma / \Gamma_{0}, \Gamma_{0}=E_{S} / N_{01}, X=\alpha^{2}, \hat{X}=\hat{\alpha}^{2}$ and $Y=\beta^{2}$. The parameter $\Gamma_{0}$ is denoted as the transmit SNR.

The random variables $X, \hat{X}$, and $Y$ follow the Gamma distribution with shape parameters $m_{X}, m_{\hat{X}}=m_{X}$, and $m_{Y}$, respectively, and have mean values $E[X]=E[\hat{X}]=\Omega_{X}=\Omega_{\hat{X}}$ and $E[Y]=\Omega_{Y}$. Their probability density function (PDF) and cumulative density function (CDF) are given by

$$
\begin{gathered}
f_{U}(x)=\left(\frac{m_{U}}{\Omega_{U}}\right)^{m_{U}} \frac{x^{m_{U}-1}}{\Gamma\left(m_{U}\right)} \exp \left(-\frac{m_{U} x}{\Omega_{U}}\right), \quad x \geq 0, \\
F_{U}(x)=1-\frac{1}{\Gamma\left(m_{U}\right)} \Gamma\left(m_{U}, \frac{m_{U} x}{\Omega_{U}}\right), \quad x \geq 0,
\end{gathered}
$$

respectively, where $U \in\{X, \hat{X}, Y\}, \Gamma(\cdot)$ denotes the Gamma function defined in $[11$, eq. $(8.310 .1)]$ and $\Gamma(\cdot, \cdot)$ the incomplete Gamma function defined in [11, eq. (8.350.2)].

Assuming the power correlation coefficient between $\alpha$ and $\hat{\alpha}$ (i.e., the correlation coefficient between $X$ and $\hat{X}$ ) is $\rho$, the joint probability distribution function (PDF) of $X$ and $\hat{X}$ is given by [9, Eq. (126)]. Thus, the joint PDF of $X$ and $\hat{X}$ is given by

$$
\begin{aligned}
f_{X \hat{X}}(x, \hat{x}) & =\left(\frac{m_{X}}{\Omega_{X}}\right)^{m_{X}+1} \frac{1}{(1-\rho) \Gamma\left(m_{X}\right)}\left(\frac{x \hat{x}}{\rho}\right)^{\frac{m_{X}-1}{2}} \\
& \times \exp \left(-\frac{m_{X}(x+\hat{x})}{(1-\rho) \Omega_{X}}\right) I_{m_{X}-1}\left(\frac{2 m_{X} \sqrt{\rho x \hat{x}}}{(1-\rho) \Omega_{X}}\right),
\end{aligned}
$$

where $I_{m_{X}-1}(\cdot)$ represents the modified Bessel function defined in [11, eq. (8.406.1)], $x \geq 0, \hat{x} \geq 0$ and $\rho \in[0,1]$.

\section{EXACT PERFORMANCE ANALYSIS}

\section{A. Outage probability}

The considered system experiences an outage when the instantaneous SNR $\gamma$ at the receiver at $D$ drops below some predefined outage threshold $\gamma_{t h}$. The outage probability is equal to the CDF of $Z$ evaluated at $\delta=\gamma_{t h} / \Gamma_{0}$, i.e.,

$$
P_{\text {out }}=F_{\gamma}\left(\gamma_{t h}\right)=F_{Z}\left(\gamma_{t h} / \Gamma_{0}\right)
$$

By following two different approaches, we derive two alternative expressions for the $\mathrm{CDF}$ of $Z$, denoted as exact $C D F I$ and exact $C D F$ II. Although given in different analytical forms, both expressions produce the same exact numerical value for a given set of input parameters.
1) Exact $C D F$ I: Based on some straightforward probabilistic theory, the CDF of $Z$ can be expressed as

$$
\begin{aligned}
\operatorname{Pr}\{Z \leq \delta\} & =\operatorname{Pr}\left\{Y \geq \frac{\delta \hat{X}}{X-\delta}, X \leq \delta\right\} \\
+ & \operatorname{Pr}\left\{Y<\frac{\delta \hat{X}}{X-\delta}, X>\delta\right\},
\end{aligned}
$$

yielding the exact CDF I,

$$
F_{Z}(\delta)=F_{X}(\delta)+\int_{\hat{x}=0}^{\infty} \int_{x=\delta}^{\infty} F_{Y}\left(\frac{\delta \hat{x}}{x-\delta}\right) f_{X \hat{X}}(x, \hat{x}) d x d \hat{x}
$$

where $F_{Y}(\cdot)$ and $f_{X \hat{X}}(\cdot)$ are given by (5) and (6), respectively.

2) Exact $C D F$ II: Alternatively to (8), the CDF of $Z$ can be expressed as

$$
\begin{aligned}
& \operatorname{Pr}\{Z \leq \delta\}=\operatorname{Pr}\left\{X \leq \delta\left(1+\frac{\hat{X}}{Y}\right)\right\} \\
& =\int_{0}^{\infty} \int_{0}^{\infty} F_{X \mid \hat{X}}\left(\delta\left(1+\frac{\hat{x}}{y}\right) \mid \hat{x}\right) f_{\hat{X}}(\hat{x}) f_{Y}(y) d \hat{x} d y \\
& =\int_{0}^{\infty} \int_{0}^{\infty} \frac{\int_{0}^{\delta(1+\hat{x} / y)} f_{X \hat{X}}(x, \hat{x}) d x}{f_{\hat{X}}(\hat{x})} f_{\hat{X}}(\hat{x}) f_{Y}(y) d \hat{x} d y
\end{aligned}
$$

Differentiation of (10) with respect to $\delta$ yields the PDF of $Z$,

$$
f_{Z}(\delta)=\int_{0}^{\infty} \int_{0}^{\infty}\left(1+\frac{u}{v}\right) f_{X \hat{X}}\left(\delta\left(1+\frac{u}{v}\right), u\right) f_{Y}(v) d u d v
$$

After a simple variable change, (11) is transformed into

$$
f_{Z}(\delta)=\int_{0}^{\infty} \int_{0}^{\infty} v(1+u) f_{X \hat{X}}(\delta(1+u), u v) f_{Y}(v) d v d u
$$

Assuming $m_{Y}$ is a positive integer, after the application of (4) and (6) in (12), the integration with respect to variable $v$ is solved by utilizing [10, Eq. (3.15.2.9)], which yields

$$
\begin{aligned}
f_{Z}(\delta) & =(1-\rho)^{m_{Y}}\left(\frac{m_{X}}{\Omega_{X}}\right)^{m_{X}-m_{Y}}\left(\frac{m_{Y}}{\Omega_{Y}}\right)^{m_{Y}} \\
& \times \frac{m_{Y} \delta^{m_{X}-1}}{\Gamma\left(m_{X}\right)} \int_{0}^{\infty} \frac{u^{m_{X}-1}(u+1)^{m_{X}}}{[u+(1-\rho) \Delta]^{m_{X}+m_{Y}}} \\
& \times L_{m_{Y}}^{m_{X}-1}\left(-\frac{m_{X} \rho \delta}{(1-\rho) \Omega_{X}} \frac{u(u+1)}{u+(1-\rho) \Delta}\right) \\
& \times \exp \left(-\frac{m_{X} \delta}{\Omega_{X}} \frac{(u+\Delta)(u+1)}{u+(1-\rho) \Delta}\right) d u
\end{aligned}
$$

where

$$
\Delta=\frac{m_{Y} \Omega_{X}}{m_{X} \Omega_{Y}}
$$

and $L_{n}^{a}(\cdot)$ is a generalized Laguerre polynomial, defined by [11, Eq. (8.970.1)]. Applying the definition of the generalized 
Laguerre polynomial in (13), we obtain

$$
\begin{aligned}
f_{Z}(\delta) & =\frac{m_{Y}(1-\rho)^{m_{Y}}}{\Gamma\left(m_{X}\right)}\left(\frac{m_{X}}{\Omega_{X}}\right)^{m_{X}-m_{Y}}\left(\frac{m_{Y}}{\Omega_{Y}}\right)^{m_{Y}} \\
& \times \sum_{n=0}^{m_{Y}}\left(\begin{array}{c}
m_{X}+m_{Y}-1 \\
m_{Y}-n
\end{array}\right)\left(\frac{m_{X} \rho}{(1-\rho) \Omega_{X}}\right)^{n} \\
& \times \frac{\delta^{m_{X}+n-1}}{n !} \int_{0}^{\infty} \frac{u^{m_{X}+n-1}(u+1)^{m_{X}+n}}{[u+(1-\rho) \Delta]^{m_{X}+m_{Y}+n}} \\
& \times \exp \left(-\frac{m_{X} \delta}{\Omega_{X}} \frac{(u+\Delta)(u+1)}{u+(1-\rho) \Delta}\right) d u .
\end{aligned}
$$

Next, we integrate (15) with respect to $\delta$ and obtain the exact CDF II,

$$
\begin{aligned}
& F_{Z}(\delta)=\frac{m_{Y}[(1-\rho) \Delta]^{m_{Y}}}{\Gamma\left(m_{X}\right)} \sum_{n=0}^{m_{Y}}\left(\begin{array}{c}
m_{X}+m_{Y}-1 \\
m_{Y}-n
\end{array}\right) \frac{1}{n !} \\
& \times\left(\frac{\rho}{1-\rho}\right)^{n} \int_{0}^{\infty} \frac{u^{m_{X}+n-1}}{(u+(1-\rho) \Delta)^{m_{Y}}(x+\Delta)^{m_{X}+n}} \\
& {\left[\Gamma\left(m_{X}+n\right)-\Gamma\left(m_{X}+n, \frac{m_{X} \delta}{\Omega_{X}} \frac{(u+\Delta)(u+1)}{u+(1-\rho) \Delta}\right)\right] d u .}
\end{aligned}
$$

Notice, exact CDF II, given by (16), is simpler to use since it requires only a single numerical integration, whereas exact $C D F$ $I$, given by (9), requires a double numerical integration. However, both CDFs will be used later in the asymptotic performance analysis of the system under different fading conditions.

\section{B. Exact average error probability}

We now derive an exact expression for the average bit-error probability (ABEP) for those modulation formats whose conditional BEP has the following analytical form:

$$
P_{E}(\gamma)=a E[Q(\sqrt{b \gamma})]
$$

where $a>0, b>0$ and $Q(x)=\frac{1}{\sqrt{2 \pi}} \int_{x}^{\infty} e^{-t^{2} / 2} d t$ is the Gaussian $Q$-function. Depending on the choice of $a$ and $b,(17)$ is applicable for binary/quadrature phase shift keying (BPSK/QPSK), frequency shift keying (FSK), and $M$-ary quadrature amplitude modulation (M-QAM). According to the technique presented in [15], the ABEP is determined by

$$
\bar{P}_{E}=\frac{a}{\sqrt{2 \pi}} \int_{0}^{\infty} F_{Z}\left(\frac{t^{2}}{b \Gamma_{0}}\right) \exp \left(-\frac{t^{2}}{2}\right) d t .
$$

Introducing (16) into (18), we obtain the following exact expression for the ABEP,

$$
\begin{aligned}
& \bar{P}_{E}=\frac{a}{2}-\frac{a m_{Y}[(1-\rho) \Delta]^{m_{Y}}}{\sqrt{2 \pi} \Gamma\left(m_{X}\right)} \sqrt{\frac{b \Gamma_{0} \Omega_{X}}{m_{X}}} \\
& \times \sum_{n=0}^{m_{Y}}\left(\begin{array}{c}
m_{X}+m_{Y}-1 \\
m_{Y}-n
\end{array}\right) \frac{\Gamma\left(m_{X}+n+1 / 2\right)}{\Gamma(n+1)}\left(\frac{\rho}{1-\rho}\right)^{n} \\
& \times \int_{0}^{\infty} \frac{u^{m_{X}+n-1}(u+1)^{-1 / 2}}{[u+(1-\rho) \Delta]^{m_{Y}-1 / 2}(u+\Delta)^{m_{X}+n+1 / 2}} \\
& \times{ }_{2} F_{1}\left(\frac{1}{2}, \frac{1}{2}+m_{X}+n ; \frac{3}{2} ;-\frac{b \Omega_{X} \Gamma_{0}}{2 m_{X}} \frac{u+(1-\rho) \Delta}{(u+\Delta)(u+1)}\right) d u,
\end{aligned}
$$

where ${ }_{2} F_{1}(\cdot)$ is the Gaussian hypergeometric function, defined in [14, Eq. (15.1.1)]. To derive (19), we combined [11, Eq. (6.4551)] and the Kummer's transformation [14, Eq. (13.1.7)] into the following identity:

$$
\begin{aligned}
& \int_{0}^{\infty} \Gamma\left(m_{X}+n, c t^{2}\right) \exp \left(-\frac{t^{2}}{2}\right) d t \\
& =\frac{\Gamma\left(m_{X}+n+1 / 2\right)}{\sqrt{c}}{ }_{2} F_{1}\left(\frac{1}{2}, \frac{1}{2}+m_{X}+n ; \frac{3}{2} ;-\frac{1}{2 c}\right) .
\end{aligned}
$$

In the special case, when both $m_{X}$ and $m_{Y}$ are arbitrary positive integers, (20) can be simplified by using [16, Eq. (07.23.03.0690.01)], in which case the integrand in (19) becomes a ratio of polynomials of the integration variable $u$.

\section{ASYMPTOTIC PERFORMANCE ANALYSIS}

In this section we concentrate on the asymptotic outage and ABEP performance, shedding some light onto the behavior of the scheme under consideration in the high SNR regime.

\section{A. Case $m_{X}>m_{Y}$}

For this case, the asymptotic analysis is based on the exact $C D F I$, given by (9), which can be alternatively written as

$$
F_{Z}(\delta)=F_{X}(\delta)+\int_{0}^{\infty} \int_{0}^{\infty} F_{Y}\left(\frac{\delta v}{u}\right) f_{X \hat{X}}(u+\delta, v) d u d v
$$

The assumption of high SNR is equivalently translated into the CDF behavior at infinitesimely low arguments. Hence, as $\delta \rightarrow 0$, (21) is transformed into

$$
F_{Z}(\delta) \approx F_{X}(\delta)+\int_{0}^{\infty} \int_{0}^{\infty} F_{Y}\left(\frac{\delta v}{u}\right) f_{X \hat{X}}(u, v) d u d v
$$

Since $\delta \rightarrow 0$, for some fixed value of $v / u$, the CDF of $Y$ that appears in the integrand of (22) can be approximated by using only the first two terms of the power series expansion of the incomplete Gamma function $\Gamma(\cdot, \cdot)$ [14, Eq. (6.5.29)], as

$$
F_{Y}(t) \sim \frac{t^{m_{Y}}}{\Gamma\left(m_{Y}+1\right)}\left(\frac{m_{Y}}{\Omega_{Y}}\right)^{m_{Y}}
$$

as $t=\delta y / x \rightarrow 0$. Using the same approximation as in (23), the first summand of (22), $F_{X}(\delta)$, can be ignored because it decays as $\delta^{m_{X}}$, and thus has a steeper slope compared to the second summand. Introducing (23) into the double integral of (22), we obtain

$$
\begin{aligned}
F_{Z}(\delta) & \sim \frac{\delta^{m_{Y}}}{1-\rho}\left(\frac{m_{Y}}{\Omega_{Y}}\right)^{m_{Y}}\left(\frac{m_{X}}{\Omega_{X}}\right)^{m_{X}+1} \frac{1}{\Gamma\left(m_{X}\right) \Gamma\left(1+m_{Y}\right)} \\
& \times \frac{1}{\rho^{\left(m_{X}-1\right) / 2}} \int_{0}^{\infty} \int_{0}^{\infty} u^{m_{Y}+\frac{m_{X}-1}{2}} v^{\frac{m_{X}-1}{2}-m_{Y}} \\
& \times \exp \left(-\frac{m_{X}(u+v)}{(1-\rho) \Omega_{X}}\right) I_{m_{X}-1}\left(\frac{2 m_{X} \sqrt{\rho u v}}{(1-\rho) \Omega_{X}}\right) d v d u
\end{aligned}
$$

as $\delta \rightarrow 0$. The above double integral is solved in closed form by utilizing [10, Eq. (3.15.2.9)] for integration with respect to variable $u$, and then utilizing [10, Eq. (3.24.1.7)] for integration 
with respect to variable $v$. After further algebraic manipulation, (24) is transformed into

$$
\begin{aligned}
F_{Z}(\delta) & \sim \frac{\delta^{m_{Y}} \Gamma\left(m_{X}-m_{Y}\right)}{\Gamma\left(m_{X}\right)}\left(\frac{\rho m_{Y}}{\Omega_{Y}}\right)^{m_{Y}} \\
& \times P_{m_{Y}}^{\left(-2 m_{Y},-m_{X}\right)}\left(1-\frac{2}{\rho}\right),
\end{aligned}
$$

as $\delta \rightarrow 0$, where $P_{n}^{(\alpha, \beta)}(\cdot)$ denotes the Jacobi polynomial, defined by $[11$, Eq. (8.960-1)]. Note that (25) can be expressed as a finite sum, and is valid when $m_{Y}$ is a positive integer.

The ideal channel estimation case is obtained by setting $\rho=1$ in (25), in which case we apply [16, Eq. (05.06.03.0003.01)]. Thus, in this case (25) reduces to $F_{Z}(\delta) \sim$ $\left(\delta m_{Y} / \Omega_{Y}\right)^{m_{Y}} / \Gamma\left(m_{Y}+1\right)$, which is a well known asymptotic expression [17, Eq. (4)].

Introducing (25) into (18) and using [11, Eq. (3.461.2)], we obtain the asymptotic ABEP for the case $m_{X}>m_{Y}$

$$
\begin{aligned}
\bar{P}_{E} & \sim\left(\frac{1}{\Gamma_{0}}\right)^{m_{Y}} \frac{a \Gamma\left(m_{X}-m_{Y}\right)\left(2 m_{Y}-1\right) ! !}{2 \Gamma\left(m_{X}\right)} \\
& \times\left(\frac{\rho m_{Y}}{b \Omega_{Y}}\right)^{m_{Y}} P_{m_{Y}}^{\left(-2 m_{Y},-m_{X}\right)}\left(1-\frac{2}{\rho}\right),
\end{aligned}
$$

which is valid for high transmit SNRs, i.e., $\Gamma_{0} \rightarrow \infty$.

\section{B. Case $m_{X}<m_{Y}$}

In this case, the asymptotic analysis is based on the exact $C D F$ $I I$, given by (16). As $\delta \rightarrow 0$, the expression in the squared brackets of the integrand in (16) is approximated similar to (23), yielding $\Gamma\left(m_{X}+n\right)-\Gamma\left(m_{X}+n, t\right) \sim t^{m_{X}+n} /\left(m_{X}+n\right)$. Introducing this approximation into (16), we notice that $\delta$ has the smallest exponent for the summation index $n=0$ (i.e., $\delta^{m_{X}}$ ), whereas the rest of the summation terms decay at steeper slopes with respect to $\delta$. Thus,

$$
\begin{aligned}
F_{Z}(\delta) & \sim \frac{\Gamma\left(m_{X}+m_{Y}\right) \Delta^{m_{Y}}(1-\rho)^{m_{Y}}}{\Gamma\left(m_{X}\right) \Gamma\left(m_{X}+1\right) \Gamma\left(m_{Y}\right)} \\
& \times\left(\frac{\delta m_{X}}{\Omega_{X}}\right)^{m_{X}} \int_{0}^{\infty} \frac{u^{m_{X}-1}(1+u)^{m_{X}}}{(x+(1-\rho) \Delta)^{m_{X}+m_{Y}}} d u .
\end{aligned}
$$

The above integral can be solved in closed form by using [11, Eq. (3.197-5)], valid when $m_{Y}$ is a positive integer. If $m_{X}$ is also a positive integer, in the integrand of (27) we apply the binomial formula to the term $(1+x)^{m_{X}}$ and use [11, Eq. (3.1943)], yielding

$$
\begin{aligned}
F_{Z}(\delta) & \sim\left(\frac{\delta m_{X}}{\Omega_{X}}\right)^{m_{X}} \frac{\Gamma\left(m_{X}+m_{Y}\right)}{\Gamma\left(m_{X}\right) \Gamma\left(m_{X}+1\right) \Gamma\left(m_{Y}\right)} \\
& \times \sum_{n=0}^{m_{X}}\left(\begin{array}{c}
m_{X} \\
n
\end{array}\right)[(1-\rho) \Delta]^{n} B\left(m_{X}+n, m_{Y}-n\right)
\end{aligned}
$$

as $\delta \rightarrow 0$, where $B(\cdot, \cdot)$ is the Beta function defined by $[14$, Eq. (6.2.2)]. Using [14, Eq. (15.4.1)], (29) can be further simplified as

$$
\begin{aligned}
F_{Z}(\delta) & \sim \frac{\delta^{m_{X}}}{\Gamma\left(m_{X}+1\right)}\left(\frac{m_{X}}{\Omega_{X}}\right)^{m_{X}} \\
& \times{ }_{2} F_{1}\left(-m_{X}, m_{X}, 1-m_{Y},(1-\rho) \Delta\right),
\end{aligned}
$$

as $\delta \rightarrow 0$, where $\Delta$ is given by (14). We note that (29) is valid when $m_{X}$ and $m_{Y}$ are arbitrary positive integers.
The ideal channel estimation case at the relay is obtained by setting $\rho=1$ in (29), in which case we apply [16, Eq. (07.23.03.0001.01)]. Thus, (29) reduces to $F_{Z}(\delta) \sim$ $\left(\delta m_{X} / \Omega_{X}\right)^{m_{X}} / \Gamma\left(m_{X}+1\right)$, which is a well known asymptotic expression [17, Eq. (4)].

Applying (29) in (18) and using [11, Eq. (3.461.2)], we obtain the asymptotic ABEP for the case $m_{X}<m_{Y}$

$$
\begin{aligned}
\bar{P}_{E} & \sim\left(\frac{1}{\Gamma_{0}}\right)^{m_{X}} \frac{a\left(2 m_{X}-1\right) ! !}{2 \Gamma\left(m_{X}+1\right)}\left(\frac{m_{X}}{b \Omega_{X}}\right)^{m_{X}} \\
& \times{ }_{2} F_{1}\left(-m_{X}, m_{X}, 1-m_{Y},(1-\rho) \Delta\right),
\end{aligned}
$$

which is valid for high transmit SNRs, i.e., $\Gamma_{0} \rightarrow \infty$.

\section{Case $m_{X}=m_{Y}=m$}

In this case, the asymptotic analysis is based on the PDF of the $C D F$ form II, given by (15). The argument of the exponential function that appears in the integrand of (15) is rewritten as

$$
-\frac{m \delta}{\Omega_{X}} u-\frac{m \delta}{\Omega_{X}}\left(1+\frac{\rho \Delta(u+1)}{u+(1-\rho) \Delta}\right) .
$$

The term in parenthesis in (31) is bounded as

$$
\begin{aligned}
\min \left\{1+\rho \Delta, \frac{1}{1-\rho}\right\} & \leq 1+\frac{\rho \Delta(u+1)}{u+(1-\rho) \Delta} \\
& \leq \max \left\{1+\rho \Delta, \frac{1}{1-\rho}\right\},
\end{aligned}
$$

which means that the second term in (31) diminishes as $\delta \rightarrow 0$. Thus, the exponential function in (15) can be approximated as $\exp \left(-m \delta u / \Omega_{X}\right)$, and the resulting integral is solved in closed form as

$$
\begin{array}{r}
\sum_{k=0}^{m+n}\left(\begin{array}{c}
m+n \\
k
\end{array}\right) \int_{0}^{\infty} \frac{u^{m+n+k-1}}{[u+(1-\rho) \Delta]^{2 m+n}} e^{-\frac{m \delta u}{\Omega_{X}}} d u \\
=\sum_{k=0}^{m+n}\left(\begin{array}{c}
m+n \\
k
\end{array}\right) \Gamma(m+n+k)\left[\frac{(1-\rho) \Omega_{X}}{\Omega_{Y}}\right]^{k-m} \\
U\left(m+n+k, k-m+1 ; \frac{m(1-\rho) \delta}{\Omega_{Y}}\right),
\end{array}
$$

where we utilized the binomial formula and [10, Eq. (2.1.3.1)]. In (33), $U(a ; b ; z)$ is the Tricomi confluent hypergeometric function [14, Eq. (13.2.5)], which for $\delta \rightarrow 0$ can be approximated according to [14, Eqs. (13.5.6)-(13.5.9)].

Using (33) in (15), we obtain a double sum, where the index of the first sum, $n$, runs from 0 to $m$ and the index of the second sum, $k$, runs from 0 to $m+n$. At first glance, one could consider neglecting the summation terms for values of $n$ from 1 to $m$, because they decay as $\delta^{m+n-1}$ whereas the remaining term (for $n=0$ ) decays as $\delta^{m-1}$. However, considering the approximation [14, Eq. (13.5.6)], we notice that, for $n>0$ and values of $k$ between $m+1$ and $m+n$, parameter $\delta$ appears as $\delta^{m+n-2}$, $\delta^{m+n-3}, \ldots, \delta^{m-1}$. Thus, the smallest exponent of $\delta$ appears for $k=m+n$, and, for each $n>0$, a single term of the other sum should be kept in the approximation $(k=m+n)$. Consequently, 
(15) is approximated for $\delta \rightarrow 0$ as

$$
\begin{aligned}
& f_{Z}(\delta) \sim \frac{m \delta^{m-1}}{\Gamma(m)}\left(\frac{(1-\rho) m}{\Omega_{Y}}\right)^{m}\left\{\left(\begin{array}{c}
2 m-1 \\
m
\end{array}\right) \sum_{k=0}^{m} \Gamma(m+k)\right. \\
& \times\left(\begin{array}{c}
m \\
k
\end{array}\right)\left[\frac{(1-\rho) \Omega_{X}}{\Omega_{Y}}\right]^{k-m} U\left(m+k, k-m+1 ; \frac{(1-\rho) m \delta}{\Omega_{Y}}\right) \\
& \left.+\sum_{n=1}^{m}\left(\begin{array}{c}
2 m-1 \\
m-n
\end{array}\right) \frac{1}{n}\left(\frac{\rho}{1-\rho}\right)^{n}\right\} .
\end{aligned}
$$

For the remaining Tricomi functions in (34), we apply [14, Eqs. (13.5.8) and (13.5.9)], yielding

$$
\begin{aligned}
f_{Z}(\delta) & \sim \frac{m \delta^{m-1}}{\Gamma(m)}\left(\frac{(1-\rho) m}{\Omega_{Y}}\right)^{m} \\
& \times\left\{( \begin{array} { c } 
{ 2 m - 1 } \\
{ m }
\end{array} ) \left[\log \left(\frac{\Omega_{Y}}{(1-\rho) m \delta}\right)-\psi(2 m)-2 \mu\right.\right. \\
& \left.+\sum_{k=0}^{m-1}\left(\begin{array}{c}
m \\
k
\end{array}\right) \frac{\Gamma(m+k) \Gamma(m-k)}{\Gamma(2 m)}\left(\frac{\Omega_{Y}}{(1-\rho) \Omega_{X}}\right)^{m-k}\right] \\
& \left.+\sum_{n=1}^{m}\left(\begin{array}{c}
2 m-1 \\
m-n
\end{array}\right) \frac{1}{n}\left(\frac{\rho}{1-\rho}\right)^{n}\right\}
\end{aligned}
$$

where $\psi(\cdot)$ is the digamma function, defined in [14, Eq. (6.3.1)], and $\mu$ is Euler's constant. The asymptotic CDF of $Z$ is obtained from (35) as

$$
\begin{aligned}
& F_{Z}(\delta) \sim \frac{\delta^{m}}{\Gamma(m)}\left(\frac{(1-\rho) m}{\Omega_{Y}}\right)^{m} \\
& \times\left\{( \begin{array} { c } 
{ 2 m - 1 } \\
{ m }
\end{array} ) \left[\frac{1}{m}+\log \left(\frac{\Omega_{Y}}{(1-\rho) m \delta}\right)-\psi(2 m)-2 \mu\right.\right. \\
& \left.+\sum_{k=0}^{m-1}\left(\begin{array}{c}
m \\
k
\end{array}\right) \frac{\Gamma(m+k) \Gamma(m-k)}{\Gamma(2 m)}\left(\frac{\Omega_{Y}}{(1-\rho) \Omega_{X}}\right)^{m-k}\right] \\
& \left.+\sum_{n=1}^{m}\left(\begin{array}{c}
2 m-1 \\
m-n
\end{array}\right) \frac{1}{n}\left(\frac{\rho}{1-\rho}\right)^{n}\right\}
\end{aligned}
$$

Applying (36) in (18) and using [11, Eq. (4.352.1)], we obtain the asymptotic ABEP for the case $m_{X}=m_{Y}=m$, which is omitted here due to space limitations.

\section{NUMERICAL EXAMPLES}

Fig. 1 depicts the average ABEP for BPSK modulation versus the transmit SNR, for the case of symmetric $S-R$ and $R-D$ channels, i.e., $\Omega_{X}=\Omega_{Y}$. The Nakagami- $m$ shape parameter was assumed to be identical for both links, emphasizing on the cases of $m_{X}=m_{Y}=1$ and $m_{X}=m_{Y}=3$. The curves were generated using (19), except for the ideal CSI case of $\rho=1$ where [13, Eq. (9)] was used, and confirmed by simulations which are not shown here for the sake of clarity. It is observed that the case of outdated CSI at the relay, which results in $\rho$ values which are smaller than one, entails a considerable performance degradation. It is worth pointing out, however, that such degradation is reflected in a shift of the ABEP curves, yet without affecting their slopes.

For $m_{X} \neq m_{Y}$ and $0 \leq \rho<1,(25),(26),(29)$, and (30) reveal that the slope of the outage and ABEP curves remains unchanged compared to the ideal case $(\rho=1)$, which implies a diversity gain that equals $\min \left\{m_{X}, m_{Y}\right\}$, regardless of $\rho$.

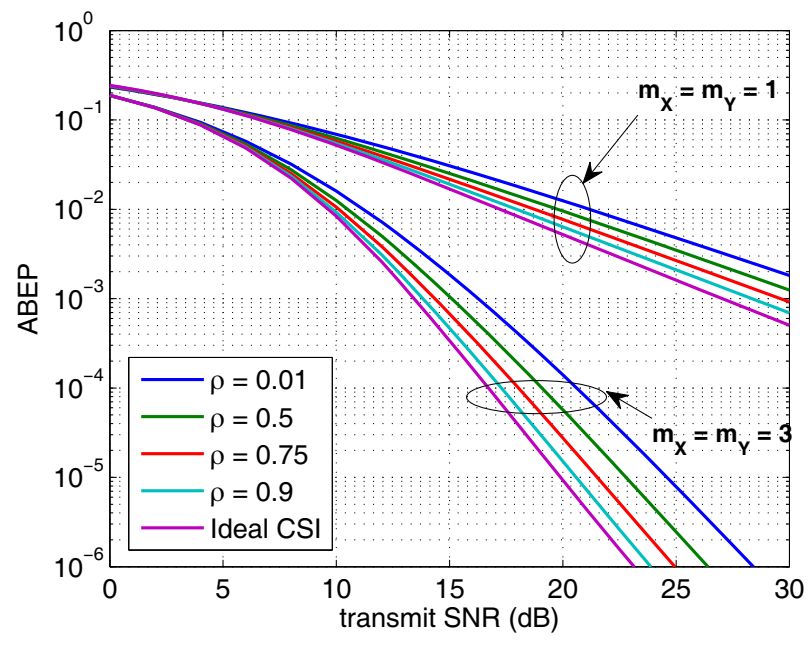

Fig. 1. ABEP for BPSK modulation vs. transmit SNR, when $\Omega_{X}=\Omega_{Y}$ (Ideal CSI curve, $\rho=1$, is obtained by using [13, Eq. (9)]).

(a) Outage probability $\left(\gamma_{t h}=1\right)$

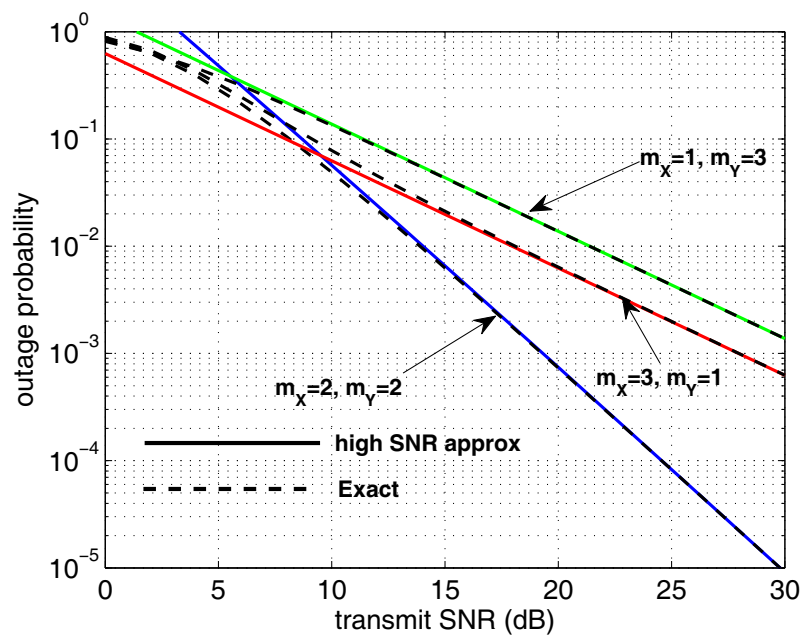

(b) ABEP (BPSK)

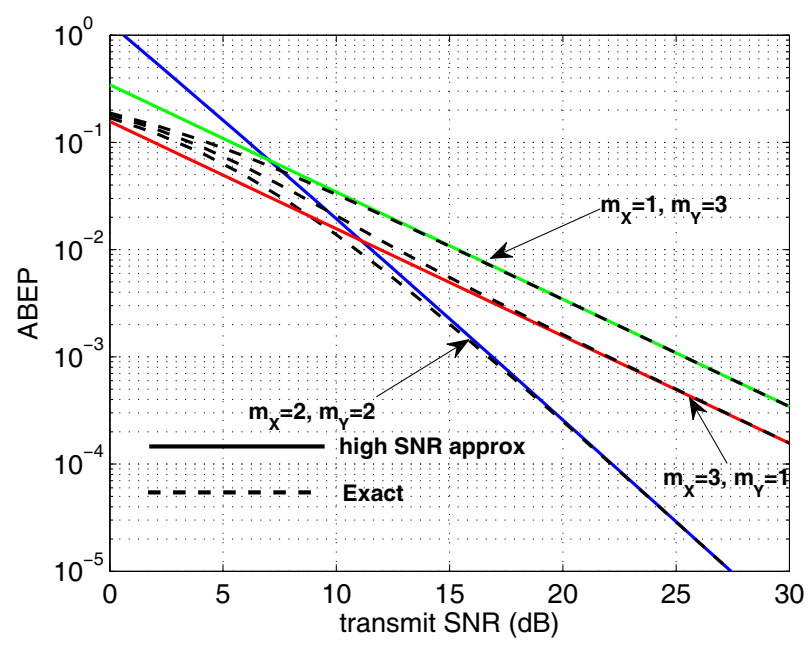

Fig. 2. Asymptotic vs. exact performance, when $\Omega_{X}=1, \Omega_{Y}=2$ and $\rho=0.5$. 


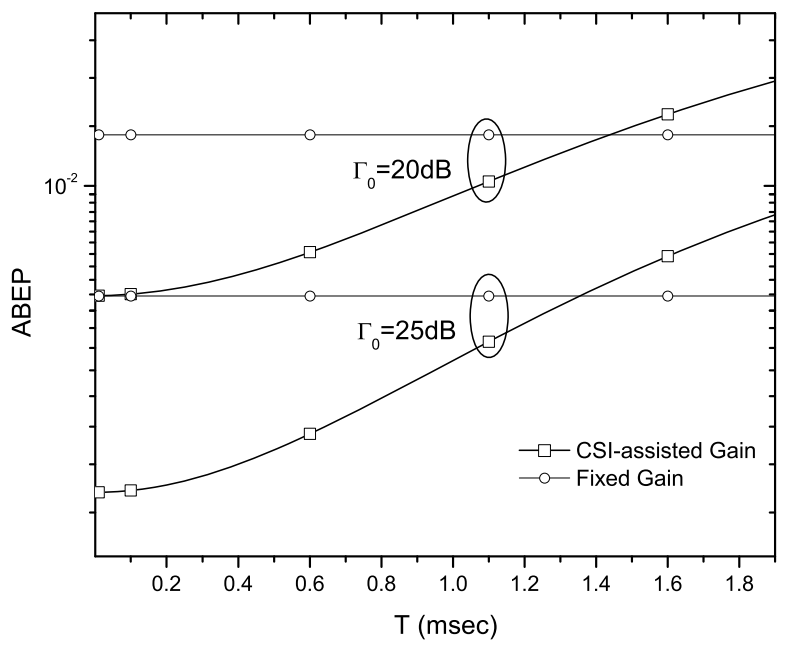

Fig. 3. ABEP for BPSK modulation vs. the update time interval of the estimation process, assuming a moving source at the speed of $50 \mathrm{~km} / \mathrm{h}$, a carrier frequency of $2.4 \mathrm{GHz}, m_{X}=m_{Y}=1, \Omega_{X}=\Omega_{Y}$, for variable-gain relaying and fixed-gain relaying. (The curve for fixed-gain relaying was generated from [19, Eq. (10)]. For a fair comparison, the gain of the fixed-gain scheme was normalized such that the average relay transmit power is the same as that of CSI-assisted gain relaying.)

For $m_{X}=m_{Y}$ and $0 \leq \rho<1$, both the outage and error probabilities decay asymptotically as $\log \left(\Gamma_{0}\right) / \Gamma_{0}^{m}$ (c.f. (36)). However, for $m_{X}=m_{Y}$ and $\rho=1$, the performance decays as $1 / \Gamma_{0}^{m}$, which is consistent with [13, Eq. (9)].

In a similar context, Figs. 2a and $2 \mathrm{~b}$ illustrate the outage and ABEP (BPSK) performance, respectively, of the scheme under consideration, for $\Omega_{X}=1, \Omega_{Y}=2, \rho=0.5$, and several combinations of $m_{X}$ and $m_{Y}$. Both the exact and the high SNR approximations, derived in Sections III and IV, respectively, are presented, showing a close match for sufficiently high SNR. The combinations of $m_{X}$ and $m_{Y}$ were chosen such that $m_{X}+m_{Y}=$ 4, i.e., $m_{X}=1, m_{Y}=3 ; m_{X}=3, m_{Y}=1 ; m_{X}=2, m_{Y}=2$.

Let us now consider a more practical aspect of the effect of outdated CSI on the performance of CSI-assisted relaying. In particular, we deal with the case where a moving source terminal at the speed of $50 \mathrm{~km} / \mathrm{h}$ transmits at a carrier frequency of 2.4 $\mathrm{GHz}$, resulting in a maximum Doppler frequency of $f_{d} \approx 100$ $\mathrm{Hz}$. The corresponding correlation coefficient between $X$ and $\hat{X}$ is obtained via Jakes' model [18] as $\rho=J_{0}^{2}\left(2 \pi f_{d} T\right)$, where $f_{d}$ is the maximum Doppler frequency, $T$ is the time interval between consecutive estimation updates and $J_{0}(\cdot)$ denotes the zeroth order Bessel function of the first kind [11, Eq. (8.411)].

Pertaining to the above scenario, Fig. 3 illustrates the ABEP of CSI-assisted relaying versus $T$, manifesting a considerable performance degradation for relatively high values of $T$. In this respect, Fig. 3 also depicts the corresponding ABEP of the socalled fixed-gain relaying scheme [19], [20], which sets a fixed gain value that depends only on the statistics of the $S$ - $R$ link [20], i.e., no instantaneous CSI is needed. We note that, due to imperfect CSI at the relay, the average relay transmit power is not the same as if perfect CSI is available. For this reason, we have normalized the gains of fixed-gain relaying and CSI-assisted gain relaying, such that the average relay transmit power is the same for both cases. It is shown that in order for CSI-assisted relaying to outperform fixed-gain relaying, the CSI has to be updated every $1.3-1.4 \mathrm{msec}$, or less, for transmit SNRs of $20 \mathrm{~dB}-25 \mathrm{~dB}$.

\section{CONCLUSION}

When the gain used for channel compensation in CSI-assisted AF relaying applications is based on outdated CSI, the overall performance is considerably impaired. The performance degradation is the larger the lower the correlation between the actual and the estimated source-relay channel, yet such performance degradation does not affect the slope of the outage and error probability curves. It was further observed that CSI-assisted relaying requires frequent updates of the source-relay channel in order to outperform its less complex counterpart, that is, fixedgain relaying.

\section{REFERENCES}

[1] 3GPP TS36.912 V9.1.0: "Feasibility study for Further Advancement for EUTRA (LTE-Advanced)," 2010.

[2] J. N. Laneman, D. N. C. Tse, and G. W. Wornell, "Cooperative diversity in wireless networks: Efficient protocols and outage behavior," IEEE Trans. on Inform. Theory, vol. 50, no. 12, pp. 3062-3080, Dec 2004

[3] G. Amarasuriya, C. Tellambura, and M. Ardakani, "Asymptotically-exact performance bounds of AF multi-hop relaying over nakagami fading,", IEEE Trans. Commun., vol.59, no.4, pp.962-967, Apr 2011

[4] H. A. Suraweera, P. J. Smith, A. Nallanathan, and Thompson, J.S., "Amplifyand-forward relaying with optimal and suboptimal transmit antenna selection," IEEE Trans. Wireless Commun., vol. 10, no. 6, pp. 1874-1885, Jun 2011

[5] M. Gatzianas, L. Georgiadis, and G. K. Karagiannidis, "Gain adaptation policies for dual-hop nonregenerative relayed systems," IEEE Trans. Commun., vol. 55, no. 8, pp. 1472-1477, Aug. 2007

[6] D. S. Michalopoulos, H. A. Suraweera, G. K. Karagiannidis, and R. Schober, "Relay selection with outdated channel estimates," IEEE Global Communications Conference (Globecom) 2010 Miami, FL, USA.

[7] M. J. Taghiyar, S. Muhaidat and J. Liang, "On the performance of pilot symbol assisted modulation for cooperative systems with imperfect channel estimation,'IEEE Wireless Communications and Networking Conference (WCNC), 2010

[8] H. A. Suraweera, M. Soysa, C. Tellambura, and H. K. Garg, "Performance analysis of partial relay selection with feedback delay", IEEE Signal Processing Letters, vol.17, pp.531-534, Jun 2010.

[9] M. Nakagami, "The $m$-distribution-a general formula of intensity distribution of rapid fading," Statistical Methods Radio Wave Propag., vol. 40, pp. 757-768, Nov. 1962

[10] A. P. Prudnikov, Y. A. Brychkov, and O. I. Marichev, Integrals and Series, Vol. 4. Direct Laplace Transforms. Gordon and Breach Science Publishers, 1986

[11] I. S. Gradshteyn and I. M. Ryzhik, Table of Integrals, Series and Products. San Diego, CA: Academic Press, 2000

[12] A. Wojnar, "Unknown bounds on performance in Nakagami channels," IEEE Trans. Commun., vol. COM-34, no. 1, pp. 22-24, Jan. 1986

[13] S. N. Datta and S. Chakrabarti, "Unified error analysis of dual-hop relay link in Nakagami- $m$ fading channels," IEEE Commun. Letters, vol. 14, no. 10, pp. 897-899, Oct. 2010

[14] M. Abramowitz and I. A. Stegun, Handbook of Mathematical Functions with Formulas, Graphs, and Mathematical Tables, 9th ed. New York: Dover, 1970

[15] Y. Zhao, R. Adve, and T. J. Lim, "Symbol error rate of selection amplifyandforward relay systems," IEEE Commun. Lett., vol. 10, pp. 757-759, Nov. 2006

[16] http://functions.wolfram.com

[17] J. Zhang, T. Zhang, J. Huang, and R. Yuan, "ABEP of amplify-and-forward cooperation in Nakagami- $m$ fading channels with arbitrary $m$, , IEEE Trans. Commun., vol. 8, no. 9, pp. 4445-4449, Sep 2009

[18] W. C. Jakes, Microwave Mobile Communication, John Wiley \& Sons, NY, 1974

[19] R. Zhao, and L. Yang, "Performance analysis of fixed gain relaying systems in Nakagami-m fading channels", IEEE International Conference on Wireless Communications and Signal Processing, Nov 2009

[20] D. B. da Costa, and M. B. Yacoub, "Dual-hop transmissions with semi-blind relays over Nakagami- $m$ fading channels", Electronics Letters, vol.44, no.3, pp.214-216, Jan 2008 